\title{
Assessing the Effects of Administering Calcium Oxide Nanoparticles on Oral Mucosa and Tongue of Male Wister Rats
}

\author{
Bushra Habeeb Al-Maula ${ }^{1}$, Zena Wally ${ }^{2}$, Rasha Dosh ${ }^{3}$, Abtesam Imhemed Aljdaimi ${ }^{4}$, \\ Suhad Jabbar Hamed Al-Nasrawie ${ }^{5}$, Julfikar Haider ${ }^{6}$ \\ ${ }^{1}$ Scientific Center of Laser and Photonics, University of Al-Hamdaniya, Ninvah, Iraq. \\ ${ }^{2}$ Department of Prosthodontic, Faculty of Dentistry, University of Kufa, Najaf, Iraq. \\ ${ }^{3}$ Department of Anatomy and Histology, Faculty of Medicine, University of Kufa, Najaf, Iraq. \\ ${ }^{4}$ College of Dentistry and Oral Surgery, Alasmarya University, Libya. \\ ${ }^{5}$ Department of Conservative Dentistry, Faculty of Dentistry, University of Kufa, Iraq. \\ ${ }^{6}$ Department of Engineering, Manchester Metropolitan University, Manchester, UK. \\ Corresponding author. E-mail: zinah.alnuaimi@uokufa.edu.iq
}

Received: May 19, 2021; Accepted: Aug. 3, 2021; Published: Sep. 9, 2021

Citation: Bushra Habeeb Al-Maula, Zena Wally, Rasha Dosh, Abtesam Imhemed Aljdaimi, Suhad Jabbar Hamed Al-Nasrawie, and Julfikar Haider, Assessing the Effects of Administering Calcium Oxide Nanoparticles on Oral Mucosa and Tongue of Male Wister Rats. Nano Biomed. Eng., 202I, I3(3): 3II-320.

DOI: $10.5101 / \mathrm{nbe} . \mathrm{v} 13 \mathrm{i} 3 . \mathrm{p} 311-320$.

\begin{abstract}
The present study was conducted to evaluate the impact of calcium oxide-nanoparticles (CaO-NPs) administration at different concentrations on the epithelial thickness, papilla length and number of blood vessels of male Wister rats' buccal mucosa, and the epithelial thickness, filiform length and number of blood vessels of the rats' tongue. Twelve rats were used and randomly allocated into four groups: control group (untreated) and the three experimental groups were orally treated with $\mathrm{CaO}$ NPs at different doses 25,50 and $100 \mathrm{mg} / \mathrm{kg}$ of the body weight over two months. On the $61^{\text {st }}$ day, all the animals were sacrificed, and $1 \mathrm{~cm}$ of the buccal mucosa and the body of the tongue were carefully removed for histological analysis. Histological tissues were studied under a light microscope to investigate the impact of CaO-NPs administration on the oral tissues. An image processing software (Image J) was used to measure the epithelial thickness, papilla length and number of blood vessels of the rats' buccal mucosa, and the epithelial thickness, filiform length and number of blood vessels of the rats' tongue. The results showed that the $\mathrm{CaO}$ nanoparticles administration caused epithelial atrophy and decreased vascularization of buccal and tongue mucosa with all tested doses of CaO-NPs and the $100 \mathrm{mg} / \mathrm{kg}$ concentration showed the most significant effect.
\end{abstract}

Keywords: $\mathrm{CaO}$ nanoparticles, Drug administration, Oral mucosa, Tongue, Histological analysis

\section{Introduction}

Several fields including medicine, engineering, agriculture, manufacturing, information and environmental technologies have been invaded by nanotechnology through its applications in commercial products $[1,2]$. Nanometer-scale materials (1-100 $\mathrm{nm}$ ) have a larger surface area to volume ratio, which improves cellular uptake efficiency and provides unique physical properties suitable in various biomedical applications [3]. Different metal oxide nanoparticles have been synthesized in the literature such as silver [4], titanium dioxide $\left(\mathrm{TiO}_{2}\right)[5,6]$, molybdenum disulfide $\left(\mathrm{MoS}_{2}\right)$ [7, 8] and calcium-based 
materials [3, 9]. Calcium based nanoparticles have been recommended in numerous studies for medicine and dentistry due to their ability to release calcium ion which forms the main elements of human bones and teeth $[10,11]$ and plays a vital role in the functioning of living cells, and thus considered to be an essential mineral for all organisms $[12,13]$. Physical weakness, muscle cramps and dental caries are all signs of low calcium levels in the blood [14].

Calcium oxide nanoparticles ( $\mathrm{CaO}-\mathrm{NPs}$ ) have been produced in several ways [15], but mainly, they are available as conventionally prepared and commercially manufactured [2]. The effects of CaONPs on various body organs such as liver, pancreas, and skin were evaluated in the literature. These particles frequently used in drug delivery and showed antimicrobial properties against tested organisms such as Pseudomonas aeruginosa, Staphylococcus epidermidis, and Candida tropicalis [16]. Oral drug delivery system is commonly used with nanoparticles treatment due to its convenience in use, noninvasive, readily acceptable alternatives to intravenous systems [17]. The absorption of orally administered drugs is influenced by the oral mucosa and tongue tissues that have unique physiological properties such as tissue permeability, penetration barriers and permeability pathway, biopharmaceutics of buccal and sublingual absorption [18].

The underlying tissues of the oral mucosa are separated from the oral environment, by the epithelium, which is composed of two layers: the superficial stratified squamous epithelium and the deep lamina propria. The main constituents of the epithelium of keratinized oral mucosa organized in four layers are namely stratum basale, stratum granulosum, stratum spinosum, and stratum corneum [19]. In the nonkeratinized epithelium, the stratum filamentosum and the stratum distendum cap the stratum basale. Distinct phenotypes are differentiated in the oral mucosa, like lining mucosa, masticatory mucosa, and specialized mucosa $[19,20]$. Lining mucosa has a non-keratinizing squamous epithelium with a loose attachment to the adjacent structures via an elastin-rich connective tissue. It is located over movable structures such as cheeks, soft palate, lips, vestibular fornix, alveolar mucosa and the floor of the mouth. Conversely, masticatory mucosa is tightly bound to the underlying bone by dense connective tissue. It is the tough and rigid protecting cap of the hard palate and the gingiva that has a keratinized epithelium. Specialized mucosa of the dorsum of the tongue is a keratinized epithelium and it includes taste buds and lingual papillae as specialized structures [19]. Squamous epithelia has structural characteristics like cornification and stratification of the keratinocytes and specific cell-to-cell interactions to keep its function as a barrier. The flexible property is required for the buccal part and floor of the mouth in order to help in speech, chewing and swallowing. A lining mucosa that is not keratinized lines these tissues [20].

The tongue is a specialized skeletal muscle that plays a vital role in speaking, breatingh and swallowing food [21]. A sufficient number of macroscopic papillae are present on the dorsal side of the tongue. These papillae are distinct as filiform, foliate, fungiform, conical, and circumvallate papillae. Filiform papillae are the ones that have a mechanical impact without taste buds. They have a keratinized squamous epithelium layer covering the connective tissue [22].

The high degree of vascularization and lack of keratinization of oral mucosa promote the rapid absorption of drugs, by passing first hepatic metabolism and hydrolysis in the gastrointestinal tract [23] the buccal mucosa offers advantages over more traditional routes. Its relative permeability and high degree of vascularization combined with the lack of keratinization lead to the rapid absorption of drugs, bypassing the hepatic first-pass metabolism and hydrolysis in the gastrointestinal tract. Conventional small molecule drugs and biologics have been administered through this route. However, the technological requirements and challenges associated with the buccal route have restricted the number of formulations available on the market or under clinical evaluation. In this chapter, nanoparticle hydrogel composites are presented as an innovative buccal drug delivery (BDD. Many nano molecules have been administered through this route. However, one of the challenges associated with the nanoparticles is that the mucosal tissue interaction still needs to be elucidated. Most of the in vivo studies with CaO-NPs have focused on assessing the impact of these particles on the skin [24], liver, kidney and brain [25] after treatment. Histological analysis of the skin and corneal revealed areas of necrosis [24]. Also, spots of hemorrhages and degenerations were seen in kidney, liver and brain after three months of $\mathrm{CaO}$ nanoparticles treatment [25].

As can be seen from the literature, there is no clear identification of the effects of CaO-NPs on the oral mucosa. To the authors' best knowledge no previous 
studies have evaluated the direct impact of oral administration of $\mathrm{CaO}-\mathrm{NPs}$ on oral mucosa or toungue. Thus, the present study aimed to evaluate the effect of oral administration of CaO-NPs at different doses $(25,50$ and $100 \mathrm{mg} / \mathrm{kg})$ over two months on the tissue structure of the oral mucosa and tongue of Wister rats.

\section{Experimental \\ Preparation of CaO-NPs}

A solution was prepared by dissolving one and a half gram of $\mathrm{CaCl}_{2} \cdot 2 \mathrm{H}_{2} \mathrm{O}$ (BDH Chemicals Ltd Poole, England) in $50 \mathrm{~mL}$ of tridistilled water after sufficient stirring. Fifteen $\mathrm{ml}$ of $\mathrm{NaOH}(1 \mathrm{M})$ was added to the mixture to form a nano-powder suspension, which was kept for $1 \mathrm{hr}$ at $75^{\circ} \mathrm{C}$. Following the cooling to ambient temperature, centrifugation was used to separate the particles. To eliminate any contaminated materials, the particles were washed with tridistilled water and dried at $80{ }^{\circ} \mathrm{C}$. The target amount $(7.5 \mathrm{mg})$ of $\mathrm{CaO}-\mathrm{NPs}$ was added to $30 \mathrm{~mL}$ of tridistilled water and underwent $5 \mathrm{~h}$ of sonication to separate $\mathrm{CaO}-\mathrm{NPs}$ and to avoid any agglomeration of the nanoparticles. Later, the drop casting method was used to deposit $\mathrm{CaO}$ layer on a glass slab to investigate the nanoparticles' characteristics such as size and crystallinity. Structural properties of $\mathrm{CaO}-\mathrm{NPs}$ was evaluated in a previous work using X-ray diffraction, and atomic force microscopy in the Faculty of Dentistry, University of Kufa, Iraq. The size distribution of CaO-NPs was found within a range between $15 \mathrm{~nm}$ to $65 \mathrm{~nm}$. The topography of the nanoparticle was fairly regular and the average roughness was $1.84 \mathrm{~nm}$ [26]. To figure out the morphology of the CaO-NPs, a scanning electron microscopy (SEM) (Inspect S50, FEI, Netherlands) was used with an acceleration voltage of $10 \mathrm{kV}$.

\section{Animals and nanoparticle administration}

The experimental methods in this study were performed by following the legislation on the use of animals in scientific research (Directive 2010/63/eu of the European parliament and of the council of 22 September 2010) with ethical approval granted by the University of Kufa (Reference number: 3203). Twelve male Wistar albino rats weighing 150-340 gm and age ranging from one to two months were obtained from the University of Kufa to conduct this investigation. The rats were housed in clean cages at the University of Kufa and were maintained under standard laboratory conditions with a $13 \mathrm{hr}$ light/11 hr dark cycle. Distilled water and commercial food bits were employed for feeding the rats. After one week of acclimation, the suspension of $\mathrm{CaO}-\mathrm{NPs}$ dosage was administered daily to the rats for 60 days. The rats were randomly allocated into 4 groups with each group comprising of three rats $(n=3)$. The control group was treated with $0.9 \%$ normal saline only and the experimental groups were treated with $\mathrm{CaO}-\mathrm{NPs}$ suspensions of 25,50 and $100 \mathrm{mg} / \mathrm{kg}$ of the body weight. It was administered orally through the oral intubation tube for 60 consecutive days. On the $61^{\text {st }}$ day, all the rats were sacrificed to assess the effects of CaO-NPs administration on the epithelial thickness, papilla length and number of blood vessels of the rats' buccal mucosa, and the epithelial thickness, filiform length and number of blood vessels of the rats' tongues. The experimental procedure is graphically presented in Fig. 1.

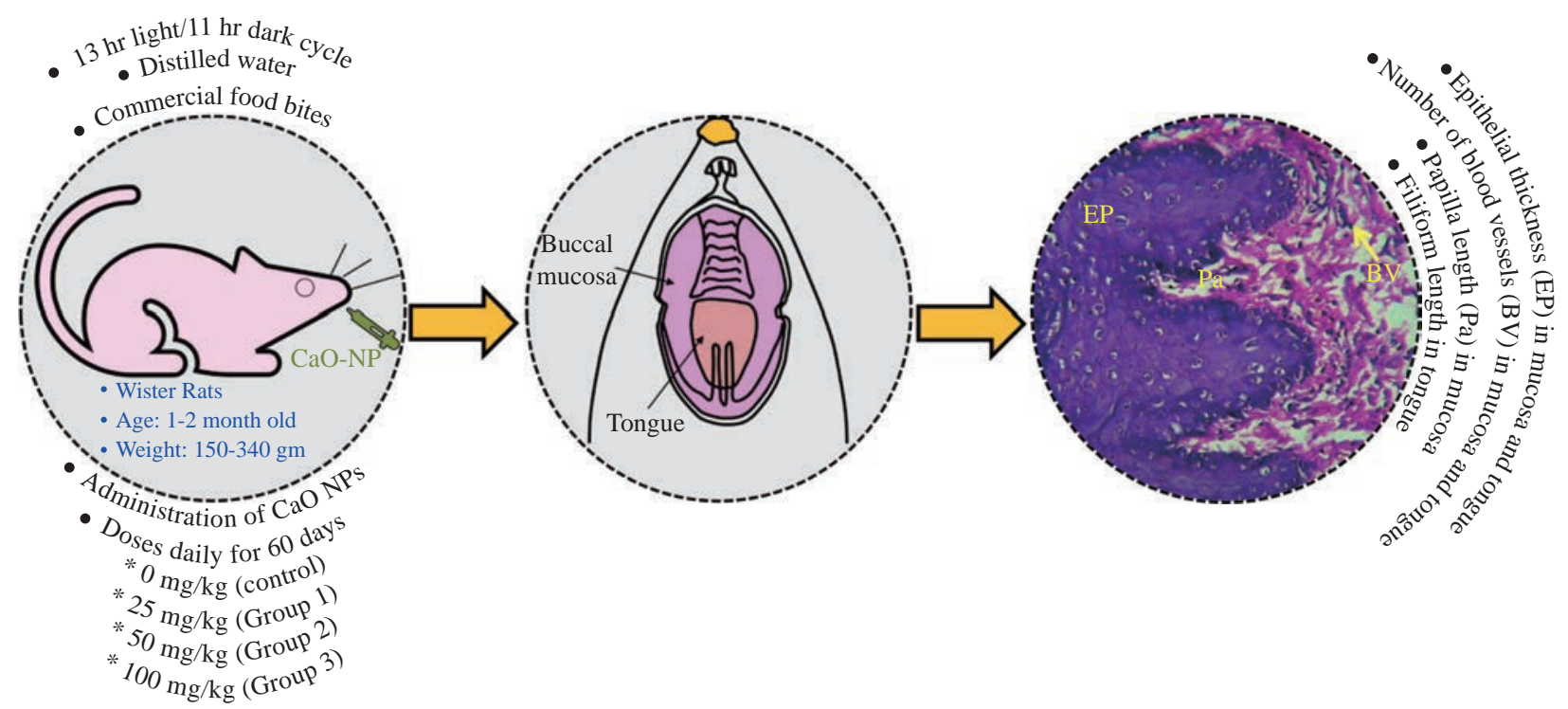

Fig. 1 Experimental procedure for evaluating the effects of $\mathrm{CaO}$ nanoparticles (NPs) administration on oral mucosa and tongue of Wister rats. 


\section{Histological preparation and measurement}

To perform histological analysis, $1 \mathrm{~cm}$ of buccal mucosa was carefully removed from the corners of the mouth of the sacrificed rats and $1 \mathrm{~cm}$ from the body of their tongue. Soft tissue specimens were immediately stored in $10 \%$ freshly prepared formalin for three days for fixation. Subsequently, the tissues were subjected to a gradual dehydration through progressively increasing alcohol percentages $(60 \%, 80 \%, 90 \%$ and absolute alcohol) for 2 hours in each stage. The specimens were passed through two changes of xylene. To remove the xylene from the tissue and to replace it with paraffin, the samples were moved to 3 successive dishes of paraffin during the course of 1-2-hours. Finally, the samples were molded in the center of paraffin blocks. Sections with a thickness of $5 \mu \mathrm{m}$ were cut using a microtome (Leittz/Germany), mounted on slides (Leica Microsystem Milton Keynes, UK), deparaffinised in xylol and rehydrated in decreasing concentrations of alcohol prior to rehydration in distilled water. The sections were then stained with Haematoxylin (Leica Microsystem, Milton Keynes, UK) for 5 mins, rinsed in water for 5 mins and finally immersed in Eosin (Leica Microsystem, Milton Keynes, UK) for 1 min. After staining, the sections were dehydrated with different grades of alcohol and cleared with xylene. Later the slides were mounted on clean glass slides in a Pertex (Leica Microsystem, Milton Keynes, UK) to allow rapid drying medium for mounting and preserve the specimens.

Histological examination of the prepared specimens was conducted by a light microscopy with a magnification of $\times 10$. Microscopical images were analyzed by Image J software (NIH, USA) to determine the epithelial thickness and papilla length of the rats' buccal mucosa and the epithelial thickness and filiform length of the rats' tongue. The epithelium >s thickness was determined from the base of the rete ridge to the surface of the keratin layer, measuring the depth of the rete ridge (papilla length). The filiform length was measured from the base of the papillae to the surface of the keratin layer in micrometers, while the blood vessels in the buccal mucosa and tongue were counted histologically by taking two fields of each slide under a light microscope using $\times 10$ magnification.

\section{Statistical analysis}

The mean and standard deviation (SD) of the obtained data are calculated. The statistical data of the experimental groups were compared to the control group. A GraphPad Prism 8 software was used to analyze the data by one-way ANOVA followed by a Tukey's multiple comparison post-hoc test (P-value $\leq$ $0.05)$.

\section{Results and Discussion Morphology of the CaO-NPs}

Fig. 2 presents a SEM image of the CaO-NPs revealing that they have semi spherical shape and quite uniform in size. However, bigger particles or clustering was formed by agglomerating many smaller particles.

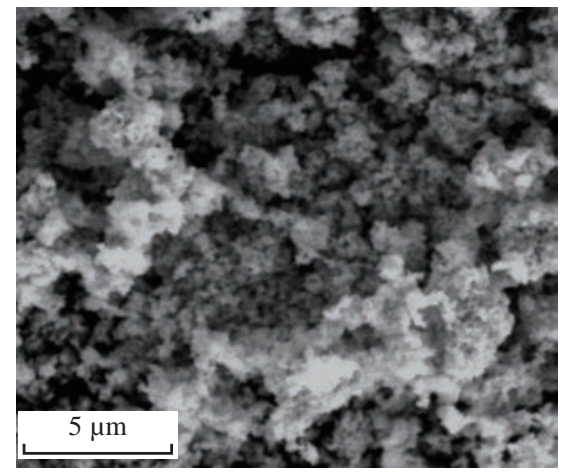

Fig. 2 SEM image of CaO-NPs.

\section{Buccal mucosa-Histological and histomorp- hometric analysis}

Histological study of Buccal mucosa showed a gradual decrease in the epithelium thickness and keratinization of the superficial layer with an increase in the dose of CaO-NPs, whereas in the control group, the non-keratinized epithelium appeared thick with numerous long and wide rete ridges, thick lamina properia and more blood vessels. Rats treated with 25 $\mathrm{mg} / \mathrm{kg}$ showed thick parakeratinized epithelium with numerous long and wide rete ridges and thick lamina properia. Again, the rats treated with $50 \mathrm{mg} / \mathrm{kg}$ showed thick keratinized epithelium with a layer of keratin. While $100 \mathrm{mg} / \mathrm{kg}$ treated group demonstrated a thin epithelium, loss of integrity of the stratified squamous layer with an irregular surface. Moreover, it showed a thin keratin layer and no rete ridges with a small number of blood vessels and a low fibre density in the thin lamina properia (Fig. 3).

Descriptive analysis of the epithelium thickness of the rats' buccal mucosa showed a significant reduction in the epithelial thickness of all CaO-NPs treated groups at different doses $(25,50,100 \mathrm{mg} / \mathrm{kg})$ compared to the untreated control group (Fig. 4(a)). The highest epithelium thickness $(690.7 \pm 102.10 \mu \mathrm{m})$ was reported for the untreated rats. While the least thickness (122.1 

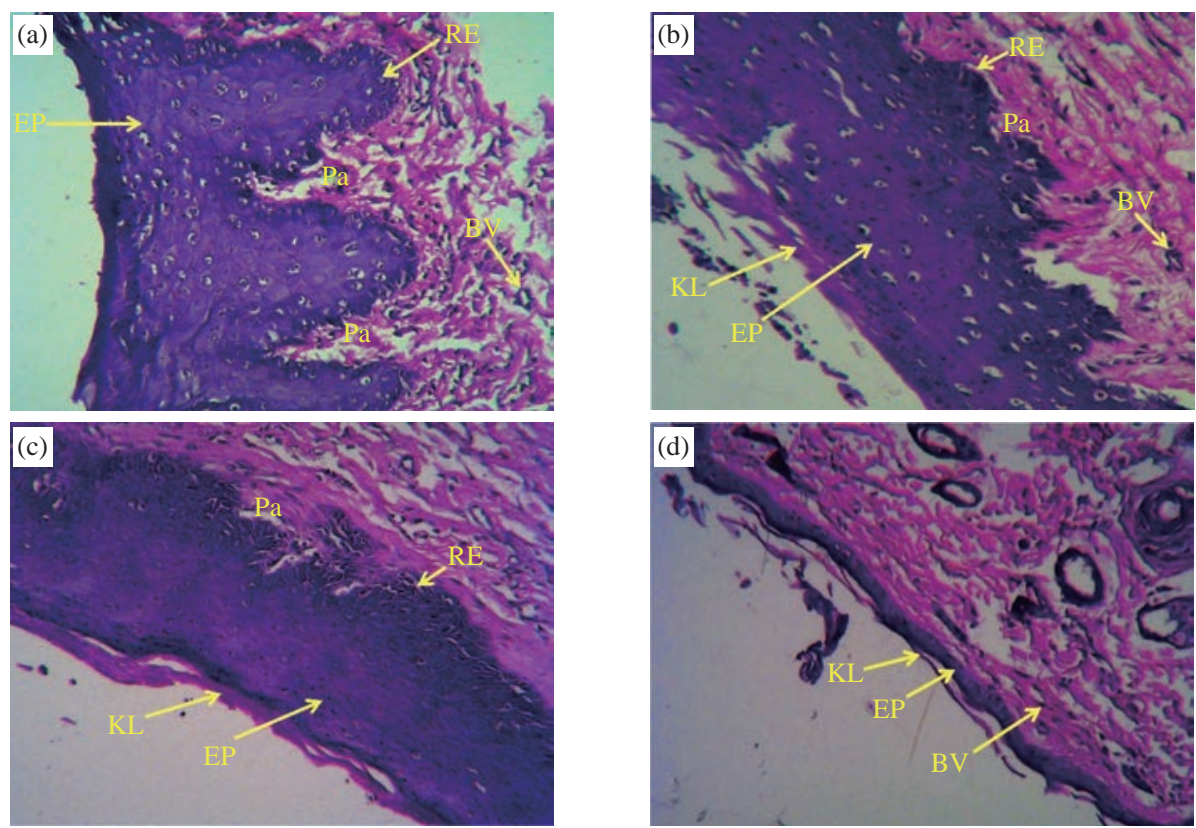

Fig. 3 Histological images of the buccal mucosa the rats under light microscope $(\times 10)$ showing (a) thick nonkeratinized epithelium in the control group, (b) thick parakeratinized epithelium in $25 \mathrm{mg} / \mathrm{kg}$ group, (c) thick keratinized epithelium in $50 \mathrm{mg} / \mathrm{kg}$ group, and (d) thin atrophic keratinized epithelium in $100 \mathrm{mg} / \mathrm{kg}$ group. (EP: epithelium; Pa: papilla; KL: keratine layer; BV: blood vessels; RE: rete ridges epithelium.)
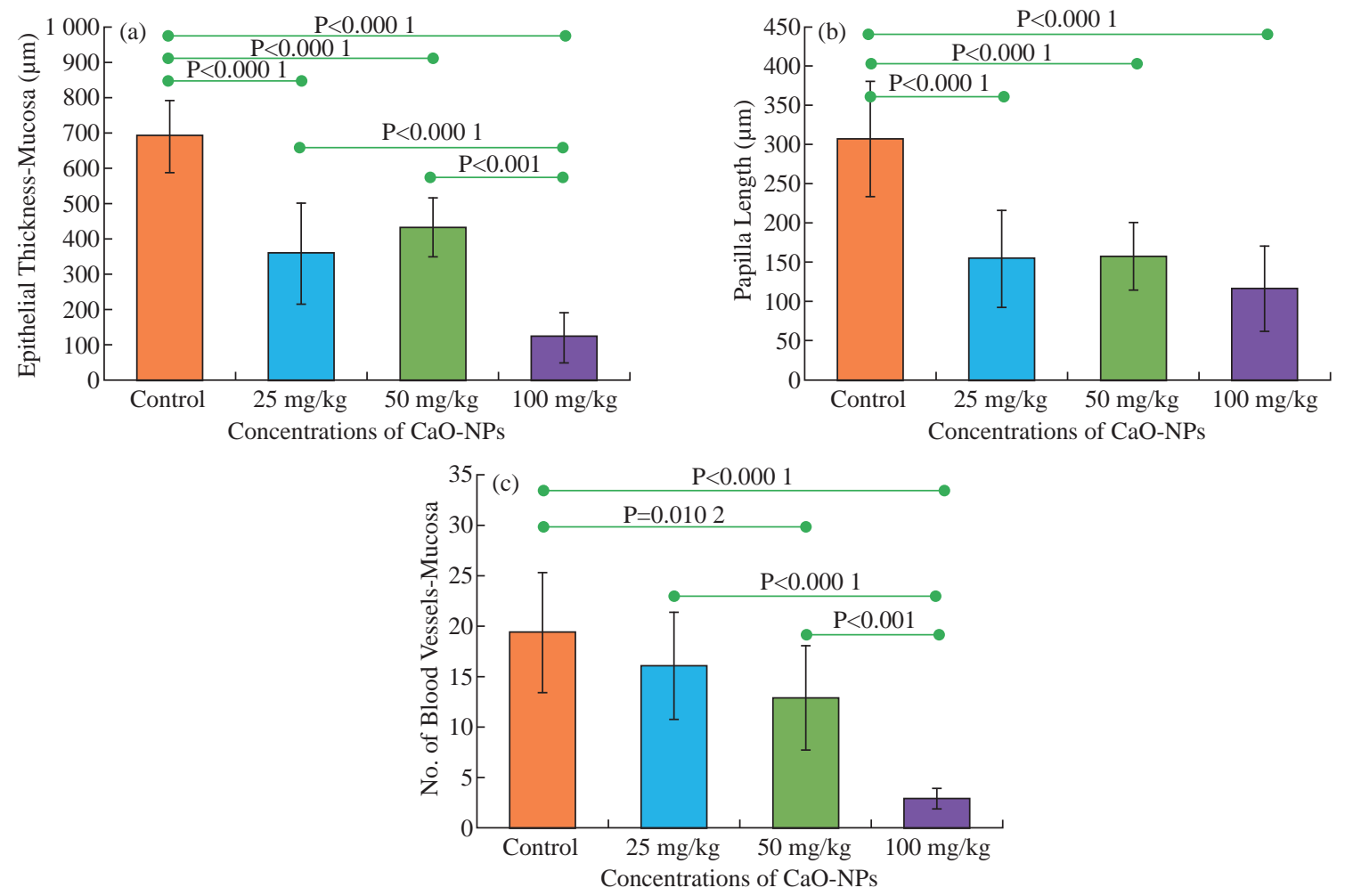

Fig. 4 Histomorphometric analysis of (a) the epithelial thickness, (b) the papilla length and (c) the number of blood vessels of the rats' buccal mucosa between the control group and the groups treated with different nanoparticle concentrations $(25,50,100 \mathrm{mg} / \mathrm{kg})$.

$\pm 69.8 \mu \mathrm{m})$ was found in rats treated with $100 \mathrm{mg} / \mathrm{kg}$ CaO-NPs. However, non-significant difference was found only between the $25 \mathrm{mg} / \mathrm{kg}$ and $50 \mathrm{mg} / \mathrm{kg}$ group.

Histological analysis of the images showed a gradual decrease in the papilla length with the increase in the dose of CaO-NPs, as shown in Fig. 4(b). The maximum length of papilla $(307.3 \pm 73.68)$ was measured for the untreated rats. This length decreased progressively to reach the least value $(116.0 \pm 55.0)$ for the rats treated with $100 \mathrm{mg} / \mathrm{kg} \mathrm{CaO}-\mathrm{NPs}$. There 
was a statistically significant difference in the papilla length of between the control group and the CaO-NPs treated groups. However, among the treated groups, no significant difference was noted.

The number of blood vessels in the lamina properia showed a progressive reduction with the increase of nanoparticle in the doses. The lowest number of blood vessels $(2.8 \pm 1.11)$ was found in the rats treated with $100 \mathrm{mg} / \mathrm{kg} \mathrm{CaO}-\mathrm{NPs}$. While the untreated rats showed the highest blood vessels number $(19.33 \pm 5.94)$ as shown in Fig. 4(c). There was a statistically significant variation in the number of blood vessels between the control group and the tested groups treated with $50 \mathrm{mg}$ / $\mathrm{kg}$ and $100 \mathrm{mg} / \mathrm{kg}$ of CaO-NPs. Furthermore, the group treated with $100 \mathrm{mg} / \mathrm{kg} \mathrm{CaO}-\mathrm{NPs}$ was significantly different from the other two treated groups.

\section{Tongue-Histological and histomorphometric analysis}

Histological study of the tongue showed a decrease in the epithelium thickness, filiform thickness and length, number of rete ridges, keratin layer thickness and the number of blood vessels gradually with the increase in the dose of $\mathrm{CaO}$ nanoparticles (Fig. 5).

Similar to the mucosa results, the epithelium thickness gradually decreased with the increasing particles does, as shown in Fig. 6(a). The untreated rats reported the highest epithelium thickness (557.9 $\pm 31.24 \mu \mathrm{m})$. Whereas the minimum thickness $(397.8$ $\pm 67.33 \mu \mathrm{m})$ was reported for the $100 \mathrm{mg} / \mathrm{kg} \mathrm{CaO}-\mathrm{NP}$ treated rats. Statistical analysis the epithelial thickness showed a significant difference between all the $\mathrm{CaO}$ NPs treated groups in comparison to the control group. However, significant differences were found only between the $50 \mathrm{mg} / \mathrm{kg}$ and $100 \mathrm{mg} / \mathrm{kg}$ treated groups.

There was a statistically significant dissimilarity in the filiform length between the untreated rats and tested groups treated with 50 and $100 \mathrm{mg} / \mathrm{kg}$ of CaONPs. This length was significantly declined from $332.3 \pm 43.24$ for the control group to the lowest value $173.6 \pm 48.47$ for the $100 \mathrm{mg} / \mathrm{kg}$ of $\mathrm{CaO}-\mathrm{NPs}$ group, as shown in Fig. 6(b). Although $25 \mathrm{mg} / \mathrm{kg} \mathrm{CaO}-\mathrm{NPs}$ treated group did not show much change from the control group, this group was significantly higher than the $50 \mathrm{mg} / \mathrm{kg}$ and $100 \mathrm{mg} / \mathrm{kg}$ groups.

The bar chart in Fig. 6(c) shows number of blood vessels in the rats' tongues from the untreated and CaO-NPs treated groups at different doses $(25,50$, $100 \mathrm{mg} / \mathrm{kg}$ ). Blood vessels count showed a significant reduction from $4.9 \pm 2.15$ at the control group to the lowest value of $1.0 \pm 0.90$ at the $100 \mathrm{mg} / \mathrm{kg} \mathrm{CaO}-$ NPs treated group. There was a statistically significant
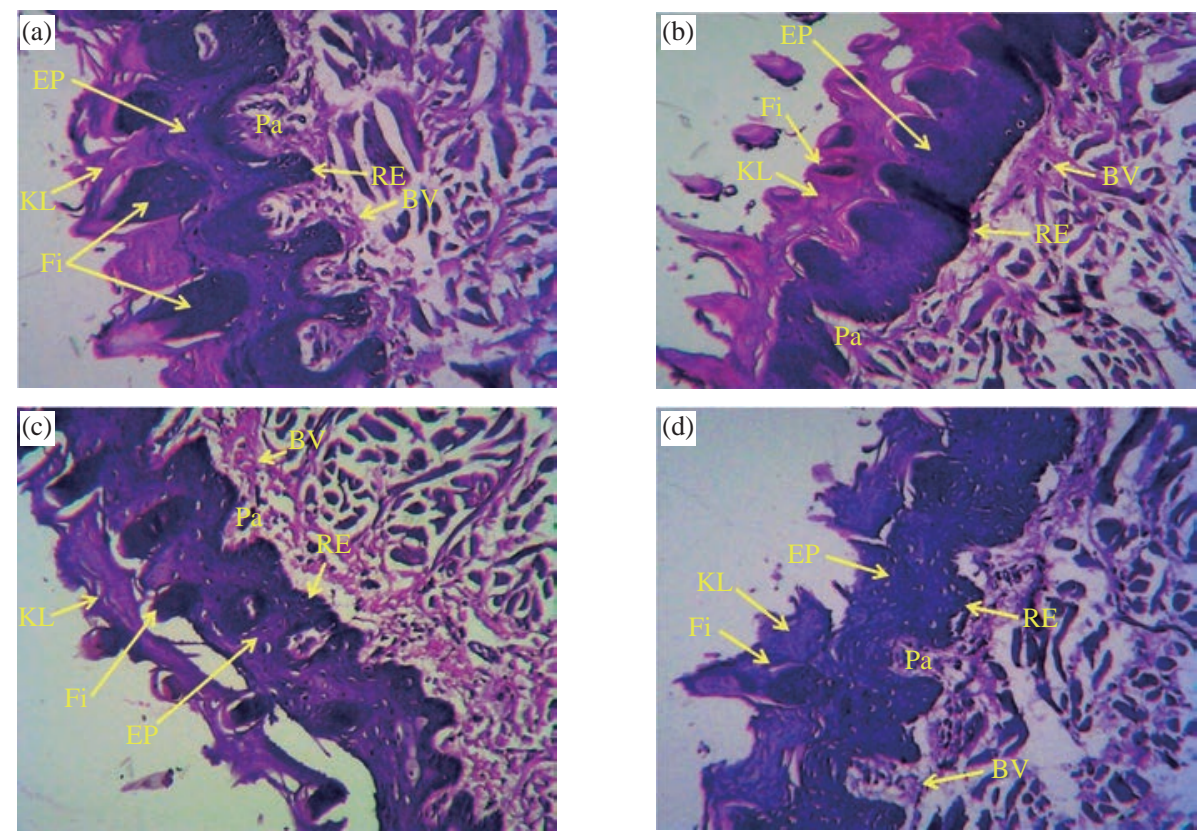

Fig. 5 Histological images of the dorsal epithelium of the rat's tongue under light microscope $(\times 10)$ showing (a) thick keratinized epithelium, large number of long rete ridges and more filiform papillae in the control group, (b) thick keratinized epithelium and reduction in the length and number of rete ridges in the $25 \mathrm{mg} / \mathrm{kg}$ group, (c) keratinized epithelium and reduction in the number of rete ridges in the $50 \mathrm{mg} / \mathrm{kg}$ group, and (d) keratinized epithelium and more reduction in the number of flat rete ridges, filiform and a thin separated keratin layer in $100 \mathrm{mg} / \mathrm{kg}$ group. (EP: epithelium; Pa: papilla; KL: keratin layer; Fi: filiform; BV: blood vessels; RE: rete ridges epithelium.). 

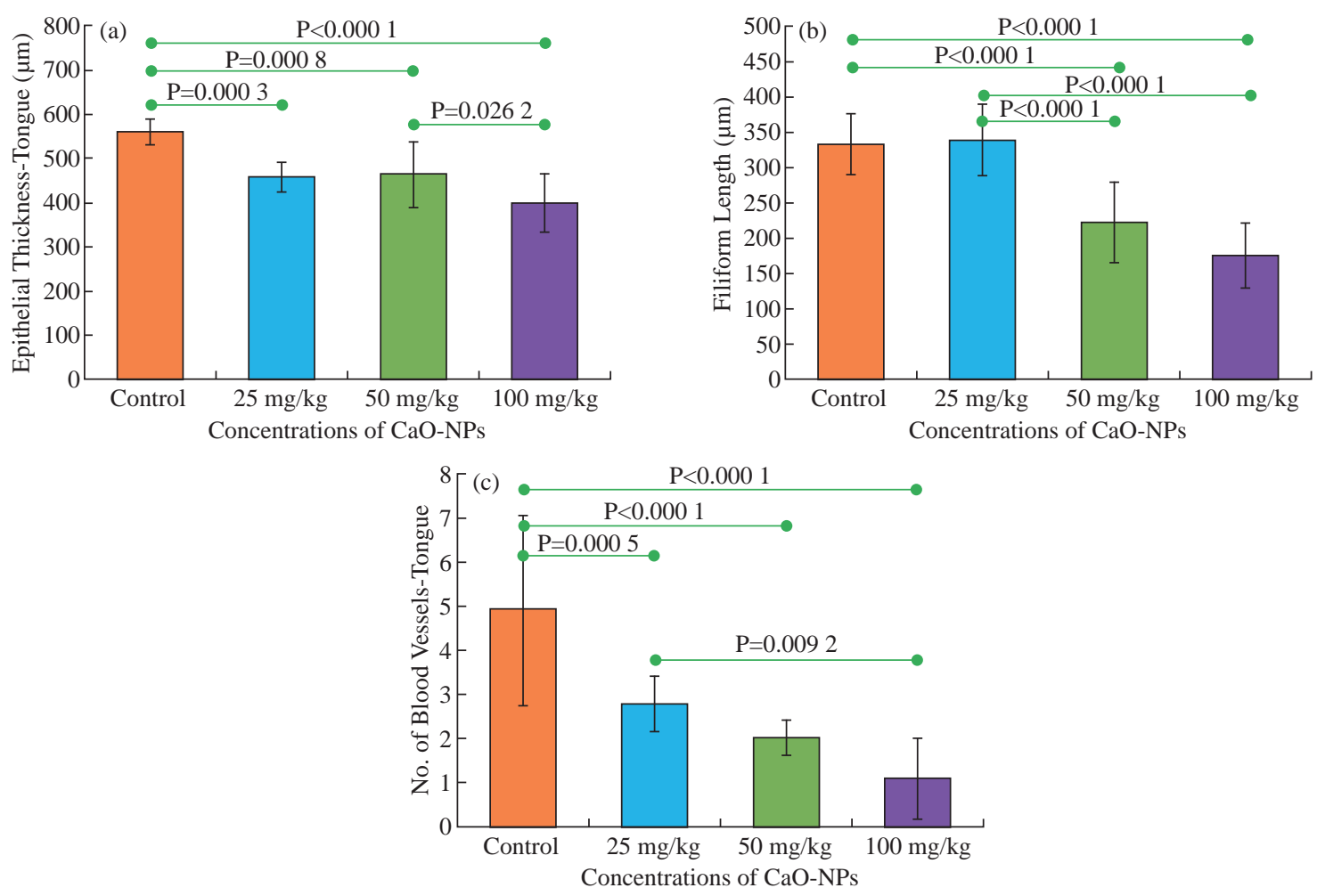

Fig. 6 Histomorphometric analysis of (a) the epithelial thickness, (b) the filiform length, and (c) the number of blood vessels of the rats' tongue between the control group and the groups treated with different nanoparticle concentrations $(25,50,100 \mathrm{mg} / \mathrm{kg})$.

reduction in the number of blood vessels between the control and all tested groups. However, among the test groups, the reduction in the number of blood vessels from $25 \mathrm{mg} / \mathrm{kg}$ to $5025 \mathrm{mg} / \mathrm{kg}$ or $5025 \mathrm{mg} / \mathrm{kg}$ to 100 $25 \mathrm{mg} / \mathrm{kg}$ was insignificant.

\section{Discussion}

The rapid invasion and high involvement of nanoparticles have increased the concern regarding human health safety, particularly in consumer products and medical applications [16, 27]. Nowadays, since nanotechnology affects our lives in many ways, understanding its safe use in healthcare applications has become a crucial issue. Nanoparticles have been efficiently utilized to increase catalysts' activity in detecting chronic diseases in the pharmaceutical industry and sensors. $\mathrm{CaO}$ is a safe material to animals and human beings owing to its biocompatibility as well as the ability to deactivate microbial endotoxin and this attracts significant attention in drug delivery [28-29] .

Although the oral cavity is the main delivery route for the nanoparticles to enter the human body through oral absorption, risk evaluation with exposure to nanoparticles uptake is still ignored. It is not clear whether nanoparticles interact with the oral mucosa or are just ingested. Thus, the present study was designed to determine the effect of of CaO-NPs administration on the tissue structure of the oral mucosa and the tongue through histological and histomorphometric analysis.

The buccal mucosa plays an important role in the drug absorption due to its permeability, lack of keratinization and considerable degree of vascularization [23]. The results in this study demonstrated that $\mathrm{CaO}-\mathrm{NPs}$ led to a reduction in the epithelium thicknesses and the length of the rete ridges in the buccal mucosa with an increase in the dose of $\mathrm{CaO}$ nanoparticles. It was observed that the epithelial thickness of both the buccal mucosa and the tongue were slightly increased at $50 \mathrm{mg} / \mathrm{kg}$ than $25 \mathrm{mg} / \mathrm{kg}$ $\mathrm{CaO}-\mathrm{NPs}$ treated groups. However, this increase was statistically insignificant; it could be probably due to the large range of data in the $50 \mathrm{mg} / \mathrm{kg}$ group. Several researchers showed that the impacts of exposure to nanoparticles on cell viability and cellular functions was dependent on the size and concentration of the nanoparticles [30-32]. The uptake of nanoparticles by cells decreased significantly as the size of nanoparticles increased [33]. In this study, the size of CaO-NPs was varied from $15 \mathrm{~nm}$ to $65 \mathrm{~nm}$ [26]. The results showed that long-term exposure (60 days) to $\mathrm{CaO}-\mathrm{NPs}$ could cause buccal mucosa and tongue tissue damage with 
an increase in the dose of $\mathrm{CaO}$ nanoparticles. This finding was in contrary to a previous study which demonstrated that no pathological changes were observed in the layers of skin (epidermis, dermis and hypodermis), whereas degenerations, hemorrhages and necrosis were observed in liver, kidney and brain post three months intravenous administration of $\mathrm{CaO}$ NPs $(11 \mathrm{~nm})$ in rats [25]. In contrast, skin burn was reported in swine after exposure to $\mathrm{CaO}$ NPs where an area of necrosis of the epidermis and dermis was observed [24].

Oral burns by chemicals occur due to chemical ingestion, leading to ulcers and burns in the oral mucosa [34]. Various kinds of drugs and chemicals can cause chemical burns whereby their severity relies on the quantity and concentration of the chemical and the pattern and extent of contact with the target tissues. However, the ingestion of corrosive materials may result in injuries with a severity varying from slight mucosal erythema to trans-mural necrosis. Redness, pain, blistering and ulceration or mucosa necrosis are usual symptoms of both thermal and chemical burns inside the mouth [35]. Saliva serves as the first line of protection against the toxic chemicals and microorganisms into the bloodstream in the oral cavity [36]. A reaction occurred when the $\mathrm{CaO}$ came into contact with the saliva (water) raising the temperature to a $100{ }^{\circ} \mathrm{C}$ within 1 minute. Furthermore, calcium hydroxide, one of the outcomes of this reaction, was highly basic, with $12.6 \mathrm{pH}$, and thus chemical burns to the mucous membranes could occur over the reaction period [37]. The pathway of nanoparticles cytotoxicity depended on apoptosis, oxidative stress, and DNA damage [38]. The calcium oxide regarded as a major factor that responsible for the reactive oxygen species (ROS) formation [16], which was the causative agent for oxidative damage in DNA of buccal mucosal cells and lastly exfoliated these cells [39].

This study illustrated that the exposure to $\mathrm{CaO}$ NPs significantly decreased the number of blood vessels in the buccal mucosa and the tongue with increasing concentrations of the nanoparticles. When nanoparticles penetrate the human body, the usual route for their distribution across the body is blood circulation. The lumen of the blood vessels is lining with the vascular endothelial cells which act as a physiological barrier that regulates the movement of the nanoparticles from the blood vessels into the tissues [40]. The integrity of the endothelial barrier is a crucial factor in evaluating nanoparticles' safety [41]. Nanoparticles can cause inflammation of the endothelial system and result in malfunction. This happens through evading from phagocytosis and directly interacting with the endothelial monolayer. Alternatively, this can happen through the phagocytosis of the nanoparticles by monocytes and causing responses to oxidative stress which induces apoptosis of endothelial cells [40]. ROS trigger hypoxia in the circulated blood which stimulates the formation of oxidant free radicals that in turn inhibit the formation of new blood vessels and destroy the junction between them [42].

It was clear from the results that both the doses of 50 and $100 \mathrm{mg} / \mathrm{kg}$ induced significant effect on all the measured parameters. However, the $25 \mathrm{mg} / \mathrm{kg}$ dose did not induce any significant effect for no of blood vessels in oral mucosa and filiform length in tongue. The highest concentration of CaO-NPs $(100 \mathrm{mg} / \mathrm{kg})$ exhibited the highest epithelial atrophy and the lowest vascularization in the buccal mucosa and tongue of the tested rats.

Even though the results obtained in this study showed the detrimental effect of the CaO-NPs on the oral mucosa, one of the limitations of this study was that histologically the buccal mucosa and tongue of the rats were not same as to that of human. ThirionDelalande et al. (2017) showed that human buccal mucosa lined with non-keratinized epithelium with large rete-ridges compared to the rat's buccal mucosa which lined with thin keratinized epithelium with smaller rete-ridges [43]. Thus, a further histological and immunohistochemical study to investigate the effect of CaO-NPs on the oral mucosa of another animal species that are similar to human is needed in the future before conducting trails on human oral mucosa.

\section{Conclusions}

The effects of regular administration of calcium oxide nanoparticles (CaO-NPs) at variable doses $(25,50$ and $100 \mathrm{mg} / \mathrm{kg})$ on the buccal mucosa and tongue of Wister rats over a period of two months was evaluated by comparing with the untreated control group. The results confirmed that $\mathrm{CaO}$ nanoparticles altered the normal structure and caused a reduction in the epithelial thickness, papilla length and number of blood vessels of the oral mucosa with an increase in the dose of $\mathrm{CaO}$ nanoparticles. A gradual reduction in the epithelium thickness, filiform thickness and length, and the number of blood vessels of the rats' tongue also confirmed a negative effect with the increase in 
the dose of CaO-NPs. Therefore, lower doses of CaONPs nanomaterials and shorter treatment periods could be recommended for future studies when administered orally to exploit the biomedical advantages of these particles.

\section{Acknowledgements}

The authors would like to acknowledge the support from University of Kufa to provide the experimental facility.

\section{Conflict of Interests}

The authors declare that no competing interest exists.

\section{References}

[1] J.W. Rasmussen, E. Martinez, P. Louka, et al., Zinc oxide nanoparticles for selective destruction of tumor cells and potential for drug delivery applications. Expert Opin. Drug Deliv., 2010, 7: 1063-1077.

[2] T.A. Robertson, W.Y. Sanchez, and M.S. Roberts, Are commercially available nanoparticles safe when applied to the skin? J. Biomed. Nanotechnol., 2010, 6: 452-468.

[3] M.D. Mauricio, S. Guerra-Ojeda, P. Marchio, et al., Nanoparticles in medicine: A focus on vascular oxidative stress. Oxid. Med. Cell. Longev. 2018, 2018.

[4] M.. Samiei, A. Farjami, S.M. Dizaj, et al., Nanoparticles for antimicrobial purposes in Endodontics: A systematic review of in vitro studies. Mater. Sci. Eng. C 2016, 58: 1269-1278

[5] A. Balati, D. Wagle, K.L Nash, et al., Heterojunction of $\mathrm{TiO}_{2}$ nanoparticle embedded into ZSM5 to 2D and 3D layered-structures of MoS2 nanosheets fabricated by pulsed laser ablation and microwave technique in deionized water: structurally enhanced photocatalytic performance. Appl. Nanosci., 2019, 9: 19-32.

[6] A. Balati, S. Tek, K. Nash, et al., Nanoarchitecture of $\mathrm{TiO}_{2}$ microspheres with expanded lattice interlayers and its heterojunction to the laser modified black $\mathrm{TiO}_{2}$ using pulsed laser ablation in liquid with improved photocatalytic performance under visible light irradiation. J. Colloid Interface Sci., 2019, 541: 234-248.

[7] A. Balati, A. Bazilio, A. Shahriar, et al., Simultaneous formation of ultra-thin $\mathrm{MoSe}_{2}$ nanosheets, Inorganic Fullerene-Like $\mathrm{MoSe}_{2}$ and $\mathrm{MoO}_{3}$ quantum dots using fast and ecofriendly Pulsed Laser Ablation in Liquid followed by microwave treatment. Mater. Sci. Semicond. Process., 2019, 99: 68-77.

[8] A. Balati, A. Matta, K. Nash, et al., Heterojunction of vertically aligned $\mathrm{MoS}_{2}$ layers to Hydrogenated Black $\mathrm{TiO}_{2}$ and Rutile Based Inorganic Hollow Microspheres for the highly enhanced visible light arsenic photooxidation. Compos. Part B Eng., 2020, 185: 107785.

[9] S. Priyadarsini, S. Mukherjee, and M. Mishra, Nanoparticles used in dentistry: A review. J. Oral Biol. Craniofacial Res., 2018, 8: 58-67.

[10] H.A. Bischoff-Ferrari, J.R. Rees, M.V. Grau, et al., Effect of calcium supplementation on fracture risk: A double- blind randomized controlled trial. Am. J. Clin. Nutr., 2008.

[11] C. Ashok, C.S. Chakra, T. Dayakar, et al., Calcium Oxide Nano Particles Synthesized From Chicken Egg Shells by Physical Method Calcium Oxide Nano Particles Synthesized From Chicken Egg Shells by Physical Method. Proceedings of International Conference on Emerging Technologies in Mechanical Sciences, 2015.

[12] H.C. Blair, L.J. Robinson, C.L.H. Huang, et al., Calcium and bone disease. BioFactors, 2011, 37: 159-167.

[13] A.B. Paula, M. Laranjo, C.M. Marto, et al., Evaluation of dentinogenesis inducer biomaterials: An in vivo study. $J$. Appl. Oral Sci., 2020, 28.

[14] P. Pravina, D. Sayaji, and M. Avinash, Calcium and its role in human body. Int. J. Res. Pharm. Biomed. Sci., 2013.

[15] Z. Mirghiasi, F. Bakhtiari, E. Darezereshki, et al., Preparation and characterization of $\mathrm{CaO}$ nanoparticles from $\mathrm{Ca}(\mathrm{OH})_{2}$ by direct thermal decomposition method. $J$. Ind. Eng. Chem., 2014, 20: 113-117.

[16] A. Roy, S.S. Gauri, M. Bhattacharya, et al., Antimicrobial activity of $\mathrm{CaO}$ nanoparticles. J. Biomed. Nanotechnol., 2013, 9: 1570-1578.

[17] D. Render, T. Samuel, H. King, et al., BiomaterialDerived Calcium Carbonate Nanoparticles for Enteric Drug Delivery. J. Nanomater., 2016, 2016.

[18] R. Bhati, R.K. Nagrajan, A Detailed Review on Oral Mucosal Drug Delivery System| International Journal of Pharmaceutical Sciences and Research. Int. J., 2012, 3: 659-681.

[19] S. Groeger, J. Meyle, Oral mucosal epithelial cells. Front. Immunol., 2019, 10: 208.

[20] L.P. Gartner, Oral anatomy and tissue types. Semin. Dermatol., 1994, 13: 68-73.

[21] M. Stone, J. Woo, J. Lee, et al., Structure and variability in human tongue muscle anatomy. Comput. Methods Biomech. Biomed. Eng. Imaging Vis., 2018, 6: 499-507.

[22] M. Kilinc, S. Erdogan, S. Ketani, et al., Morphological Study by Scanning Electron Microscopy of the Lingual Papillae in the Middle East Blind Mole Rat (Spalax ehrenbergi, Nehring, 1898). J. Vet. Med. Ser. C Anat. Histol. Embryol., 2010, 39: 509-515.

[23] S.J. Morantes, D.M. Buitrago, J.F. Ibla, et al., Composites of hydrogels and nanoparticles: A potential solution to current challenges in buccal drug delivery. In BiopolymerBased Composites: Drug Delivery and Biomedical Applications; Elsevier Inc., 2017: 107-138.

[24] J.T.S.A. Macêdo, J.A. Ferreira Júnior, K.A. Nascimento, et al., Skin burn and ocular damage by calcium oxide (virgin lime) in swines. Pesqui. Veterinária Bras., 2018, 38: 2088-2091.

[25] A.R. Butt, S. Ejaz, J.C. Baron, et al., $\mathrm{CaO}$ nanoparticles as a potential drug delivery agent for biomedical applications. Dig. J. Nanomater. Biostructures, 2015, 10: 799-809.

[26] S.W. Al-Shaibani, The effect of calcium oxidenanoparticles on the function of the kidney organ in the rats. J. Eng. Appl. Sci., 2018, 13: 7689-7692.

[27] S. Komiyama, R. Miyasaka, K. Kikukawa, et al., Can nano-hydroxyapatite permeate the oral mucosa? A histological study using threedimensional tissue models. PLoS One, 2019, 14.

[28] S. Abraham, V. Sarathy, Biomedical Applications of Calcium Oxide Nanoparticles-A Spectroscopic Study. 2018.

[29] S. Pasupathy. M. Rajamanickam, Synthesis of pure and bio modified calcium oxide (cao) nanoparticles using waste chicken egg shells and evaluation of its antibacterial activity | International Journal of Pharmaceutical Sciences And research. Int. J. Pharm. Sci. Res., 2019, 10: 47314737. 
[30] K.Y Win, S.S. Feng, Effects of particle size and surface coating on cellular uptake of polymeric nanoparticles for oral delivery of anticancer drugs. Biomaterials, 2005, 26: 2713-2722.

[31] C.D. Walkey, J.B. Olsen, H. Guo, et al., Nanoparticle size and surface chemistry determine serum protein adsorption and macrophage uptake. J. Am. Chem. Soc., 2012, 134: 2139-2147.

[32] J. Tan, S. Shah, A. Thomas, et al., The influence of size, shape and vessel geometry on nanoparticle distribution. Microfluid. Nanofluidics, 2013, 14: 77-87.

[33] Y. Liu, E. Yoo, C. Han, et al., Endothelial barrier dysfunction induced by nanoparticle exposure through actin remodeling via caveolae/raft-regulated calcium signalling. NanoImpact, 2018, 11: 82-91.

[34] S. Kang, K. Kufta, T.P. Sollecito, et al., A treatment algorithm for the management of intraoral burns: A narrative review. Burns, 2018, 44: 1065-1076.

[35] C. Gilvetti, S.R. Porter, and S. Fedele, Traumatic chemical oral ulceration: A case report and review of the literature. Br. Dent. J., 2010.

[36] E.S. Frenkel, K. Ribbeck, Salivary mucins in host defense and disease prevention. J. Oral Microbiol. 2015, 7: 29759.

[37] Y. Hagiwara, K. Seki, and Y. Takahashi, Oral chemical burn due to accidental ingestion of calcium oxide food desiccant in a patient with dementia. J. Int. Med. Res., 2020, 48.

[38] E.I. Hassanen, E.A. Morsy, A.M. Hussien, et al., The effect of different concentrations of gold nanoparticles on growth performance, toxicopathological and immunological parameters of broiler chickens. Biosci. Rep., 2020, 40.

[39] U. Nair, H. Bartsch, and J. Nair, Alert for an epidemic of oral cancer due to use of the betel quid substitutes gutkha and pan masala: A review of agents and causative mechanisms. Mutagenesis, 2004, 19: 251-262.

[40] A.B. Engin, M. Neagu, K. Golokhvast, et al., Nanoparticles and endothelium: An update on the toxicological interactions. Farmacia, 2015, 63: 792-804.

[41] Y. Liu, E. Yoo, C. Han, et al., Endothelial barrier dysfunction induced by nanoparticle exposure through actin remodeling via caveolae/raft-regulated calcium signalling. NanoImpact, 2018, 11: 82-91.

[42] D. Wulf, Free Radicals in the Physiological Control of Cell Function. Physiol. Rev., 2002, 88: 47-95.

[43] C. Thirion-Delalande, F. Gervais, C. Fisch, et al., Comparative analysis of the oral mucosae from rodents and non-rodents: Application to the nonclinical evaluation of sublingual immunotherapy products. PLoS One, 2017, 12.

Copyright $($ Bushra Habeeb Al-Maula, Zena Wally, Rasha Dosh, Abtesam Imhemed Aljdaimi, Suhad Jabbar Hamed AlNasrawie, and Julfikar Haider. This is an open-access article distributed under the terms of the Creative Commons Attribution License, which permits unrestricted use, distribution, and reproduction in any medium, provided the original author and source are credited. 